\title{
Versão brasileira do Attribution Questionnaire \\ - Adaptação transcultural e validação de propriedades psicométricas
}

\author{
Brazilian version of the Attribution Questionnaire - Cross cultural \\ adaptation and validation of psychometric properties
}

Alexandre de Araújo Pereira', Silvana Maria Elói Santos², Rosa Malena Delbone de Faria²

\section{RESUMO}

Objetivo: Realizar a adaptação transcultural do Attribution Questionnaire - AQ27 para o português falado no Brasil a partir da versão de Portugal e validar as propriedades psicométricas da escala por meio da análise fatorial exploratória. Métodos: Após a adaptação semântica e cultural, o questionário foi aplicado a 431 alunos do $1^{\circ}$ ao $6^{\circ}$ ano do Curso de Medicina Unifenas-BH, Brasil, e, em seguida, conduziu-se análise fatorial exploratória pelo método de extração de componentes principais e rotação Varimax. Resultados: A versão final brasileira do Questionário de Atribuição (AQ-26B) apresentou um fator a menos que a versão original em inglês e a portuguesa devido à fusão dos fatores Medo e Percepção de Perigo, o que gerou a supressão da questão 11 do questionário brasileiro por não apresentar valores psicométricos adequados. Dificuldades linguísticas relacionadas à interpretação da questão 11 e sua maior

\section{Palavras-chave}

Questionário, estigma social, transtornos mentais, atitude, psiquiatria comunitária.

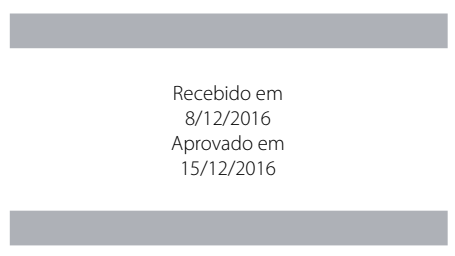

DOI: $10.1590 / 0047-2085000000139$ complexidade de significado latente parecem ter contribuído com esse resultado. Conclusões: $O$ questionário brasileiro (AQ-26B) manteve parâmetros de validade e confiabilidade adequados observando-se coerência com o modelo teórico original. Além disso, mostrou-se de fácil aplicação, demonstrando ser um instrumento útil para avaliar o estigma relacionado à doença mental entre alunos de medicina de escolas médicas brasileiras.

\section{ABSTRACT}

Objective: A cross-cultural adaptation of the Portuguese version of the Attribution Questionnaire - AQ27 for Brazilian speakers and an exploratory factor analysis were conducted in order to validate the scale's psychometric properties. Methods: After semantic and cultural adaptation of the questionnaire, exploratory factor analysis was conducted through principal component extraction and Varimax rotation methods in 431 students, from the first to the last years of the course, at Unifenas Medical Course in Belo Horizonte, Brazil. Results: The final Brazilian version of the Attribution Questionnaire (AQ-26B) had 8 factors instead of 9 from the original American and Portuguese versions. This change was due to the merger of Fear and Dangerousness factors. Question 11 was removed from the Brazilian version because it did not present adequate psychometric values. Language difficulties, related to the

1 Universidade José do Rosário Vellano (Unifenas), Curso de Medicina. 2 Universidade Federal de Minas Gerais (UFMG), Faculdade de Medicina.

Endereço para correspondência: Alexandre de Araújo Pereira Rua Professor Moraes, 562/806 31150-370 - Belo Horizonte, MG, Brasil

E-mail: alex68@uol.com.br 


\section{Keywords}

Questionnaire, social stigma, mental disorders, attitude, community psychiatry. interpretation of its meaning and its complex latent meaning, seemed to have contributed to this result. Conclusions: The Brazilian version of the Attribution Questionnaire (AQ-26B) presented suitable validity and reliability parameters as well as theoretical coherence to the original model. Moreover, it proved easy to apply, showing to be a useful tool to evaluate the stigma attached to mental illness in Brazilian medical students.

\section{INTRODUÇÃO}

Os transtornos psiquiátricos representam 14\% da carga global de doenças. Transtornos psiquiátricos mais severos, como a esquizofrenia, estão associados a taxa maior de problemas físicos de saúde e decréscimo da expectativa de vida de 12 a 15 anos, por apresentarem maior associação com obesidade, sedentarismo, tabagismo e maior risco de suicídio'.

A literatura nacional2-4 e a internacional ${ }^{5-8}$ mostram que parcelas significativas da população, incluindo profissionais de saúde, possuem visão estigmatizada dos portadores de transtornos mentais, o que pode contribuir para a exclusão social, baixa qualidade assistencial e dificuldade de acesso ao tratamento em saúde ${ }^{9-11}$. Entre alunos de medicina, vários autores identificaram atitudes predominantemente negativas ou ambivalentes em relação a essa população ${ }^{12-17}$.

Segundo Goffman 18 , "[...] o processo de estigma acontece quando um grupo de pessoas atribui uma marca discriminatória ou característica distinta a outro grupo minoritário, usualmente, privando-o de direitos e privilégios que the deveriam estar associados". Identificar o estigma entre estudantes de medicina e avaliar estratégias educacionais que promovam atitudes mais positivas na redução do estigma, por parte dos futuros médicos, pode ajudar a reverter esse cenário.

Várias escalas de mensuração do estigma foram descritas na literatura ${ }^{19-21}$. No Brasil, foram identificadas duas escalas validadas que medem atitudes e opiniões sobre as doenças mentais ${ }^{22-24}$. No entanto, esses instrumentos não avaliam diretamente o estigma, são compostos de muitos itens (51 e 70, respectivamente) e apresentam-se desatualizados.

O Attribution Questionnaire - AQ27, escala desenvolvida por Corrigan et al..25, embasada na teoria de atribuição desenvolvida por Heider ${ }^{26}$ e Weiner ${ }^{27}$, mostrou-se promissor na mensuração do estigma junto à população geral e entre profissionais de saúde. Segundo essa teoria, os indivíduos tendem a construir explicações relativas à causa e à capacidade de controle de determinado comportamento de outros indivíduos, relacionando esses aspectos com o fator responsabilidade. De acordo com o tipo de inferência produzida pelo observador, poderão ocorrer reações emocionais e, consequentemente, comportamentos de ajuda ou rejeição. Quando as pessoas consideram que a causa do comportamento alterado está sob o controle do indivíduo, portanto, que ele é responsável pelo seu estado, geralmente a reação sobre o indivíduo é negativa. Por outro lado, se a causa da doença for associada a fatores externos ao indivíduo, a atribuição de responsabilidade diminui e, consequentemente, preveem-se a diminuição das respostas emocionais negativas e o aumento dos comportamentos de ajuda. O questionário em questão descreve brevemente, em forma de vinheta, a história de um jovem portador de esquizofrenia e 27 itens exploram as atitudes, sentimentos e comportamentos dos respondentes, cujas respostas são assinaladas utilizando-se escala do tipo Likert de 1 a 9. Os itens são agrupados em nove dimensões diferentes, no original: anger, dangerousness, fear, coercion, segregation, avoidance, help, pity e responsability. Propriedades psicométricas já foram avaliadas por outros autores de língua inglesa ${ }^{28} \mathrm{e}$ italiana ${ }^{29,30}$. Uma versão preliminar portuguesa foi obtida pela tradução e retrotradução para a língua inglesa e a escala foi testada em familiares de pacientes portadores de esquizofrenia em Portuga ${ }^{31}$. No entanto, suas propriedades psicométricas, em especial a validação pela análise fatorial em português, ainda não haviam sido definidas.

O objetivo deste estudo foi realizar a adaptação transcultural do Attribution Questionnaire - AQ27VP para o português falado no Brasil e validar as propriedades psicométricas da escala por meio da análise fatorial.

\section{MÉTODOS}

\section{Procedimentos da adaptação transcultural}

Foi necessária uma adaptação transcultural do instrumento para a realidade brasileira, de acordo com as premissas defendidas por Reichenheim e Moraes ${ }^{32}$. Segundo esses autores, o processo de adaptação transcultural de um instrumento deve representar uma combinação entre a tradução literal de palavras (equivalência semântica) com um processo meticuloso de sintonização que contemple o contexto cultural e estilo de vida da população-alvo. Finalmente, devem ser conduzidos estudos psicométricos para a equivalência da validade dimensional e confiabilidade do instrumento em estudo. Numa primeira etapa, uma médica portuguesa (DPD), radicada no Brasil há muitos anos e com formação em saúde mental, sugeriu modificações da versão portuguesa. Em seguida, essas modificações foram discutidas pelo grupo de pesquisadores e incorporadas na versão brasileira. Dos 27 itens que compõem a escala, 16 não sofreram qualquer modificação, seis apresentaram apenas mudanças na estrutura da elaboração da frase e outras quatro necessitaram de 
adequação cultural semântica. Posteriormente, a versão brasileira foi testada em 24 alunos do curso de medicina, para verificação da compreensibilidade do questionário e verificação de equivalência operacional do instrumento, tais como: veículo e formato das questões, cenário de administração, modo e tempo de aplicação e categorização dos itens.

\section{Características da amostra}

Foram convidados 563 alunos de graduação, regularmente matriculados no curso de Medicina Unifenas- $\mathrm{BH}$, do $1^{\circ}$ ao $6^{\circ}$ ano, para participar deste estudo. Do total de alunos convidados, 431 concordaram em participar e autorresponderam ao questionário antes ou após atividades curriculares regulares, na presença do pesquisador responsável ou de sua equipe, devidamente treinada para esse fim. $\mathrm{O}$ estudo foi aprovado pelo Comitê de Ética em Pesquisa em Seres Humanos da Unifenas (parecer no 631.425) e todos os alunos que participaram do estudo assinaram o Termo de Consentimento Livre e Esclarecido (TCLE), como propõe a legislação em vigor ${ }^{33}$. A idade dos alunos participantes $(76,55 \%$ do total) variou de 17 a 46 anos, com média de 23,2 anos e desvio-padrão de 4,0 anos. Houve predomínio de alunos do sexo feminino $(60,8 \%)$ e solteiros $(95,4 \%)$.

\section{Procedimentos da análise fatorial exploratória}

Com o objetivo de verificar a validade de construto do AQ-27, realizou-se análise fatorial exploratória utilizando o método de extração dos componentes principais e rotação Varimax. A conveniência do modelo da análise fatorial, como técnica para redução e sumarização de variáveis, foi verificada pelo teste de esfericidade de Bartlett e pela medida de adequacidade da amostra de Kaiser-Meyer-Olkin (KMO). Ela mostrou-se conveniente no que tange aos resultados que avaliam a sua adequacidade, os autovalores e o percentual de variância explicada (Tabela 1). O coeficiente alfa de Cronbach foi a medida utilizada para avaliar a confiabilidade da consistência interna de cada um dos fatores determinados pela análise fatorial. Por fim, a determinação da composi-

Tabela 1. Avaliação dos parâmetros da análise fatorial para o modelo final do questionário de atribuição de estigma em relação ao portador de doença mental (AQ-26B)

\begin{tabular}{lcc}
\hline Parâmetros de adequacidade & Valores ideais & $\begin{array}{c}\text { Valores observados } \\
\text { no estudo }\end{array}$ \\
\hline KM0 (MSA total) & $\geq 0,60$ & 0,86 \\
Teste de esfericidade de Bartlett (p) & $<0,05$ & $<0,0001$ \\
MSA (questões individuais) & $\geq 0,50$ & $0,58-0,94$ \\
Alpha de Cronbach - fatores & $\geq 0,50$ & $0,33-0,92$ \\
Autovalores dos fatores & $>1,0$ & $1,37-5,00$ \\
Total da variância explicada - 8 fatores (\%) & $\geq 50 \%$ & $68,9 \%$ \\
Carga fatorial das questões & $>0,40$ & $0,52-0,91$ \\
Comunalidade & $\geq 0,50$ & $0,49-0,88$ \\
\hline
\end{tabular}

ção de cada um dos fatores foi baseada nas cargas fatoriais e, neste estudo, com base no tamanho da amostra, no nível de significância (5\%) e no poder de $80 \%$, considerou-se como carga fatorial significante um valor de referência, em módulo, superior a 0,35. Questões com sentido inverso ao conjunto predominante das questões avaliadas tiveram sua pontuação invertida. Isso ocorreu nos fatores Desamparo (F2) e Afastamento (F4).

\section{RESULTADOS}

\section{Adaptação transcultural}

Dos 27 itens que compõem a escala, 16 não sofreram qualquer modificação. Dos 11 restantes, seis apresentaram apenas mudanças na estrutura da elaboração da frase, usualmente pouco utilizada no Brasil. Por exemplo, Q1 - Eu iria sentir-me incomodado pelo José para Eu me sentiria incomodado por José. Outras quatro necessitaram de uma adequação cultural semântica, já que os termos existiam no português falado no Brasil, mas poderiam gerar algum grau de confusão interpretativa. Por exemplo, Q26 - Se eu fosse senhorio, provavelmente alugaria um apartamento ao José para Se eu fosse o proprietário, provavelmente alugaria um apartamento para José. Por último, em apenas uma questão, houve necessidade de troca da palavra por outra equivalente pela inexistência da palavra no português falado no Brasil. Por exemplo, Q16 Eu partilharia uma boleia de carro com o José, todos os dias para Eu pegaria uma carona de carro com José, todos os dias.

\section{Análise fatorial}

Inicialmente, verificou-se a fatoriabilidade da matriz de correlação entre os itens da escala por meio do índice Kaiser-Meyer-Olkin (KMO), obtendo-se o valor 0,86 para a amostra estudada. A análise fatorial exploratória, utilizando o método dos componentes principais e rotação Varimax, indicou oito fatores com autovalores maiores do que 1,00, explicando 68,9\% da variância total (Tabela 2). Os questionários originais de 27 itens, versão original em inglês (AQ-27) e a portuguesa (AQ-27VP), foram comparados aos da versão brasileira de 26 itens (AQ-26B). As versões de 27 itens eram distribuídas em nove fatores (três itens por fator). O modelo gerado pelo estudo atual gerou oito fatores, havendo redistribuição de alguns itens do questionário entre os fatores identificados, descritos no Quadro 1. Para facilitar a compreensão do público brasileiro e adequar melhor a interpretação dos fatores, após a redistribuição dos itens, houve modificação de nomes de alguns dos fatores. Dessa forma, todos os fatores passaram a apresentar uma mesma direção conceitual, na qual, quanto maior o escore de cada fator, maior o estigma: Anger/ Irritação passou a ser chamado de Intolerância; Avoidancel Evitamento foi definido como Afastamento; Help/Ajuda teve 
seu sentido convertido para Desamparo. Todos os itens relacionados ao fator Dangerousness/Perigosidade foram reagrupados no fator Fear/Medo, que também incorporou a Q (questão) 1, originalmente relacionada ao fator Anger/ Irritação. A Q25, originalmente relacionada ao fator Coercion/ Coação, foi incorporada ao fator Segregação. A Q-27, originalmente presente no fator Pity/Pena, foi reagrupada em
Desamparo. A questão 11 foi excluída desta análise por apresentar um baixo valor de comunalidade $(0,29)$. E, apesar de a questão de no 1 apresentar um valor de comunalidade pouco abaixo do recomendado $(0,49)$, ela foi mantida na análise, uma vez que sua retirada impactou sensivelmente no total da variância explicada pelo modelo. Além disso, sua carga fatorial foi expressiva $(0,65)$.

Tabela 2. Cargas fatoriais, média (desvio-padrão), comunalidade ( $\left.h^{2}\right)$ e medidas da adequacidade da amostra baseadas na Análise de Componentes principais com rotação VARIMAX em relação às 26 questões do questionário de Estigma (AQ-26B)

\begin{tabular}{|c|c|c|c|c|c|c|c|c|c|c|c|}
\hline \multirow[b]{2}{*}{ Questões } & \multicolumn{11}{|c|}{ Análise de componentes principais } \\
\hline & $F_{1}$ & $F_{2}$ & $F_{3}$ & $F_{4}$ & $\mathbf{F}_{5}$ & $\mathrm{~F}_{6}$ & $\mathbf{F}_{7}$ & $\mathrm{~F}_{8}$ & $\begin{array}{l}\text { Média } \\
\text { (dp) }\end{array}$ & $h^{2}$ & MSA \\
\hline 1. Eu me sentiria incomodado por José. & 0,65 & & & & & & & & $3,9(2,3)$ & 0,486 & 0,90 \\
\hline 2. Eu me sentiria inseguro perto de José. & 0,81 & & & & & & & & $4,1(2,2)$ & 0,693 & 0,92 \\
\hline 3. 0 José iria assustar-me. & 0,82 & & & & & & & & $3,3(2,1)$ & 0,723 & 0,92 \\
\hline 4. Até que ponto ficaria zangado com José? & & & & & & 0,87 & & & $2,4(1,7)$ & 0,849 & 0,79 \\
\hline 5. Se eu fosse responsável pelo tratamento de José, pediria para ele tomar a medicação. & & & & & & & & 0,83 & $8,3(1,4)$ & 0,720 & 0,71 \\
\hline 6. Penso que José coloca a sua vizinhança em risco se não for internado. & & & 0,52 & & & & & & $3,2(2,2)$ & 0,538 & 0,92 \\
\hline 7. Se eu fosse um empregador, entrevistaria José para um emprego. (INV) & & & & 0,75 & & & & & $5,7(2,6)$ & 0,637 & 0,84 \\
\hline 8. Eu estaria disposto a conversar com José sobre seus problemas. (INV) & & 0,65 & & & & & & & $7,3(2,1)$ & 0,566 & 0,85 \\
\hline 9. Eu sentiria pena de José. & & & & & 0,90 & & & & $4,2(2,6)$ & 0,874 & 0,69 \\
\hline 10. Eu pensaria que José é o culpado da sua situação atual. & & & & & & & 0,83 & & $1,3(1,0)$ & 0,700 & 0,58 \\
\hline 12. Até que ponto se sentiria irritado com José? & & & & & & 0,85 & & & $2,6(1,7)$ & 0,845 & 0,79 \\
\hline 13. Até que ponto sentiria que José é perigoso? & 0,74 & & & & & & & & $3,9(1,9)$ & 0,679 & 0,94 \\
\hline $\begin{array}{l}\text { 14. Até que ponto concorda que José deveria ser forçado a tratar-se com o seu médico } \\
\text { mesmo que ele não quisesse? }\end{array}$ & & & & & & & & 0,70 & $5,8(2,6)$ & 0,621 & 0,87 \\
\hline $\begin{array}{l}\text { 15. Eu penso que seria melhor para a comunidade onde José está inserido se ele fosse } \\
\text { colocado num hospital psiquiátrico. }\end{array}$ & & & 0,80 & & & & & & $2,5(2,0)$ & 0,742 & 0,86 \\
\hline 16. Eu pegaria uma carona de carro com José, todos os dias. (INV) & & & & 0,73 & & & & & $4,0(2,6)$ & 0,602 & 0,86 \\
\hline $\begin{array}{l}\text { 17. Até que ponto acha que um hospício, onde José pudesse estar afastado da sua } \\
\text { vizinhança, seria o melhor local para ele? }\end{array}$ & & & 0,74 & & & & & & $2,0(1,6)$ & 0,644 & 0,87 \\
\hline 18. Eu me sentiria ameaçado por José. & 0,79 & & & & & & & & $3,0(1,9)$ & 0,738 & 0,92 \\
\hline 19. Até que ponto sentiria medo de José? & 0,85 & & & & & & & & $3,2(1,9)$ & 0,790 & 0,89 \\
\hline 20. Até que ponto estaria disposto a ajudar José? (INV) & & 0,84 & & & & & & & $7,8(1,6)$ & 0,776 & 0,75 \\
\hline 21. Até que ponto tem a certeza de que iria ajudar José? (INV) & & 0,82 & & & & & & & $6,8(1,9)$ & 0,720 & 0,75 \\
\hline 22. Até que ponto sentiria pena de José? & & & & & 0,91 & & & & $3,8(2,5)$ & 0,884 & 0,68 \\
\hline 23. Até que ponto acha que José é responsável pela sua situação atual? & & & & & & & 0,81 & & $2,1(1,8)$ & 0,674 & 0,63 \\
\hline 24. Até que ponto você se sentiria assustado por José? & 0,81 & & & & & & & & $3,3(1,8)$ & 0,741 & 0,93 \\
\hline $\begin{array}{l}\text { 25. Se eu fosse responsável pelo tratamento de José, iria forçá-lo a viver numa moradia } \\
\text { protegida para pessoas com transtornos mentais. }\end{array}$ & & & 0,73 & & & & & & $2,2(1,8)$ & 0,602 & 0,89 \\
\hline 26. Se eu fosse o proprietário, provavelmente alugaria um apartamento para José. (INV) & & & & 0,73 & & & & & $5,7(2,7)$ & 0,581 & 0,85 \\
\hline 27. Até que ponto me preocuparia com José? (INV) & & 0,66 & & & & & & & $6,4(2,0)$ & 0,500 & 0,85 \\
\hline Autovalor & 5,0 & 2,4 & 2,4 & 1,9 & 1,9 & 1,7 & 1,4 & 1,4 & & & \\
\hline Porcentagem de Variância Explicada & 19,2 & 9,1 & 9,1 & 7,1 & 7,1 & 6,5 & 5,5 & 5,3 & & $\rightarrow$ Tot & $=68,9 \%$ \\
\hline Alfa de Cronbach & 0,92 & 0,74 & 0,76 & 0,66 & 0,89 & 0,84 & 0,49 & 0,33 & & & \\
\hline
\end{tabular}

MSA: Medida da Adequacidade da Amostra (Measures of Sampling Adequacy). h²: Comunalidade. Fatores: $F_{1}$ (Medo); $F_{2}$ (Desamparo); $F_{3}\left(\right.$ Segregação); $F_{4}$ (Afastamento); $F_{5}$ (Pena); $F_{6}$ (Intolerância); $F_{3}($ Responsabilidade) e $F_{8}$ (Coerção). INV: indica que a nota da questão foi invertida, p. ex. Q7_INV = 10-07. Medidas de avaliação da adequacidade para a aplicação da Análise Fatorial: KM0: 0,86e Teste de esfericidade de Bartlett: $p<0,0001$. Devido a0 baixo poder de discriminação (cargas fatoriais muito baixas), a questão de número 11 foi retirada para a composição dos índices, ou seja, foi considerada irrelevante. 
Quadro 1. Distribuição das questões do questionário em relação aos fatores, considerando as versões $A Q-27, A Q-27 V P$ e $A Q-26 B$

\begin{tabular}{|c|c|c|c|c|c|}
\hline$A Q-27 / A Q-27 V P$ & Fatores/Interpretação & Questões & $A Q-26 B$ & Fatores/Interpretação & Questōes \\
\hline Fear/Medo & $\begin{array}{l}\text { Pessoas com TM provocam } \\
\text { medo nas pessoas }\end{array}$ & Q3, Q19, Q24 & Medo (F1) & $\begin{array}{l}\text { Pessoas com TM provocam } \\
\text { medo nas pessoas porque } \\
\text { são imprevisíveis e violentas }\end{array}$ & $\begin{array}{l}\text { Q1, Q2, Q3, Q13, Q18, Q19, } \\
\text { Q24 }\end{array}$ \\
\hline Help/Ajuda & $\begin{array}{l}\text { Pessoas com TM precisam de } \\
\text { assistência }\end{array}$ & $Q 8,020,021$ & Desamparo (F2) & $\begin{array}{l}\text { Pessoas com transtornos } \\
\text { mentais não merecem ajuda }\end{array}$ & $Q 8, Q 20,021, Q 27$ \\
\hline Segregation/Segregação & $\begin{array}{l}\text { Pessoas com TM devem } \\
\text { ser encaminhadas para } \\
\text { instituições fora da } \\
\text { comunidade }\end{array}$ & Q6, Q15, Q17 & Segregação (F3) & Idem & Q6, Q15, Q17, Q25 \\
\hline Avoidance/Evitamento & $\begin{array}{l}\text { Não desejo conviver com } \\
\text { pessoas com TM }\end{array}$ & $Q 7,016,026$ & Afastamento (F4) & Idem & $07,016,026$ \\
\hline Pity/Pena & $\begin{array}{l}\text { Pessoas com TM são tomadas } \\
\text { pela sua doença, merecendo } \\
\text { preocupação e pena }\end{array}$ & Q9, Q22, Q27 & Pena (F5) & Idem & Q9, Q22 \\
\hline Anger//rritação & $\begin{array}{l}\text { Pessoas com TM são } \\
\text { culpadas por terem sua } \\
\text { doença e provocarem raiva } \\
\text { em outras pessoas }\end{array}$ & Q1, 04,012 & Intolerância (F6) & Idem & Q4, 012 \\
\hline $\begin{array}{l}\text { Responsability/ } \\
\text { Responsabilidade }\end{array}$ & $\begin{array}{l}\text { Pessoas com TM podem } \\
\text { controlar seus sintomas e } \\
\text { são responsáveis pela sua } \\
\text { doença }\end{array}$ & Q10, Q11, Q23 & Responsabilização (F7) & Idem & $Q 10,023$ \\
\hline Coercion/Coação & $\begin{array}{l}\text { Pessoas com TM devem se } \\
\text { submeter ao tratamento }\end{array}$ & Q5, Q14, Q25 & Coerção (F8) & Idem & 05,014 \\
\hline $\begin{array}{l}\text { Dangerousness/ } \\
\text { Perigosidade }\end{array}$ & $\begin{array}{l}\text { Pessoas com TM são } \\
\text { imprevisíveis e violentas }\end{array}$ & $Q 2,013,018$ & - & - & - \\
\hline
\end{tabular}

A Q11 foi retirada do modelo do estudo.

AQ-27 - Original em inglês; AQ-27VP - original em português; AQ-26B - versão brasileira. Em negrito são destacadas as questões que mudaram de fator ou construto; F: fator.

\section{DISCUSSÃO}

Kogan e Shea ${ }^{34}$ apontaram a Análise Fatorial e a definição do alfa de Cronbach como os métodos mais frequentemente utilizados na validação de escalas no campo da educação médica. A validade de testes ou escalas constitui um parâmetro da medida tipicamente discutida nas ciências sociais. Validade, segundo Pasquali35, é entendida como a propriedade de um teste estar de fato se referindo (representando) um fator, ou seja, se está medindo aquilo que se propõe a medir. Já o conceito de validação é mais complexo, porque se trata de provar cientificamente a validade de um instrumento. Também segundo Pasqualiib isto é particularmente o caso nos enfoques que, em psicologia, trabalham com o conceito de traço latente, em que se deve demonstrar a correspondência (congruência) entre traço latente e sua representação física (o comportamento). A Teoria de Resposta ao Item (TRI), que embasa essa perspectiva da psicometria moderna, define a qualidade dos testes (que são comportamentos ou variáveis observáveis) em função de um critério que não é o comportamento e sim variáveis hipotéticas, as quais chama de traço latente, também conhecido por outros nomes, como: fator, construto, conceito, estrutura psíquica, traço cognitivo, atitude, dentre outros.
Portanto, a elaboração de instrumentos, escalas dentro da TRI, depende de procedimentos teóricos: desenvolvimento da teoria psicológica sobre esse traço e a elaboração dos comportamentos que os representam (itens) por experts; procedimentos empíricos: definição de uma amostra representativa e aplicação dos itens a esses sujeitos para os quais o teste está sendo construído; procedimento analítico: demonstração da adequação do modelo aos dados empíricos, por meio da análise fatorial.

Outro fator de grande relevância na discussão metodológica da elaboração de escalas é a definição de fidedignidade, confiabilidade ou precisão de um teste. Significa que o mesmo teste, medindo os mesmos sujeitos em ocasiões diferentes, ou testes equivalentes, medindo os mesmos sujeitos na mesma ocasião, produzem resultados idênticos, isto é, a correlação entre essas duas medidas deve ser igual a 1. Entretanto, como sempre existem fatores perturbadores ou confounding variables em todas as experiências científicas, o erro está sempre presente em qualquer medida ${ }^{37}$. Uma das técnicas de estimar a precisão de um teste em uma única aplicação é por meio da análise da consistência interna dos itens, isto é, verificando a congruência que cada item do teste tem com o restante dos itens do mesmo teste. Assim, quanto menos variabilidade um mesmo item produz numa 
amostra de sujeitos, menos erros ele provocará. Uma análise estimativa da confiabilidade do teste pode ser efetuada pela fórmula de Cronbach, mediante seu coeficiente alfa: quanto mais próximo de 1, mais homogêneos são os itens entre si e maior é a consistência interna do teste, ou seja, mais preciso ele conseguirá medir aquilo que ele se propõe a medir ${ }^{35}$.

A Análise Fatorial conduzida pelo presente estudo (Tabelas 1 e 2 e Quadro 1), que validou o AQ-26B, apresentou cargas fatoriais altas (todas acima de 0,65 - com exceção da Q6 = 0,52), o que caracterizou uma correlação forte de cada variável (questão) com o fator correspondente (validade de construto). No que se refere à confiabilidade da consistência interna, que avalia o grau de congruência de cada item do teste com o restante dos itens do mesmo teste (precisão da medida), a versão brasileira (AQ-26B) apresentou alfa de Cronbach geral para a escala de 0,83 semelhante aos estudos portugueses - Sousa et al. ${ }^{31}$, Marques et al. ${ }^{38}$ e Barrantes ${ }^{39}$, respectivamente 0,88, 0,76, 0,83 e italianos Pingani et al. ${ }^{30}$ e Pingani et al. ${ }^{29}$, respectivamente 0,68 e 0,81. No entanto, quando considerados os fatores isoladamente, o AQ-26B apresentou valores baixos de confiabilidade interna para Responsabilidade (F7) e Coerção (F8), respectivamente, 0,49 e 0,33. Esse achado coincidiu com dois estudos portugueses e um italiano. Em Sousa et al. ${ }^{31}$, os resultados foram de 0,13 (Responsabilização) e 0,21 (Coerção), em Marques, Barbosa e Queirós ${ }^{38}$ foram de 0,42 (Responsabilização) e 0,37 (Coerção) e em Pingani et al. ${ }^{30}$ foram de 0,48 (Responsabilização) e 0,63 (Coerção). Os dois últimos incluíram alunos das áreas da saúde, o que pode ter contribuído para um resultado mais parecido com o encontrado em nosso estudo.

Brown ${ }^{28}$ também aplicou uma análise fatorial exploratória do AQ-27, versão original em inglês, com o intuito de estudar mais detalhadamente suas propriedades psicométricas. O autor estudou 677 alunos de psicologia de uma instituição de ensino americana e concluiu que uma solução de seis fatores representava uma variância total explicada de 65,29\%, um pouca abaixo dos 68,9\% encontrados nesse estudo. Os valores de confiabilidade avaliados pelo alfa de Cronbach para cada um dos seis fatores analisados variaram de 0,60 a 0,93 , sendo o mais baixo relativo ao fator Responsabilidade e o mais alto relacionado aos fatores Medo/Percepção de Perigo. A Q11 (Até que ponto acha que é controlável a causa da situação atual de José?), que está originalmente relacionada ao fator Responsability ${ }^{25}$, foi retirada do AQ-26B. Como descrito nos resultados, observou-se um baixo valor de comunalidade $(0,29)$ na Q11. Esse resultado sugere que os alunos não compreenderam claramente o significado desse item da escala. Durante o piloto, realizado após a adaptação transcultural da tradução brasileira, esta foi a única questão que suscitou dúvida de compreensão entre os alunos, confirmada pela análise fatorial. Aparentemente, é mais difícil elabo- rar itens relacionados à ideia de responsabilizar o portador de transtorno mental pela causa da sua doença, em inglês - responsability. A complexidade do fator e as dificuldades linguísticas relacionadas à expressão clara do significado latente indicam maior dificuldade de mensuração desse fator. Isso não foi identificado nos fatores Intolerância e Pena, que, apesar de serem representados por apenas duas questões, não apresentaram dificuldade de compressão por parte dos alunos estudados. A reformulação do item responsability, na língua portuguesa, ou mesmo a inclusão de mais itens que tragam o mesmo traço latente implícito no questionário original, poderia contribuir com a melhora da confiabilidade da consistência interna desse item, o que acarretaria em uma melhora dos parâmetros psicométricos da escala como um todo. O Fator Coerção, originalmente Coercion (inglês)/Coacção (português de Portugal), apresentou uma mudança do número de itens, com a perda da Q25 para o fator Segregação. Isso pode ter contribuído com os parâmetros psicométricos encontrados posteriormente para esse fator. Não obstante esses achados, optou-se por manter esses dois fatores (Responsabilização e Coerção) no AQ-26B por representarem uma percentagem importante da variância explicada (10,8\%), além de estarem coerentes com os pressupostos teóricos que embasaram a escala origina ${ }^{25}$. Por outro lado, o fator Medo apresentou os maiores valores de alfa de Cronbach nas versões já validadas em inglês e italiano. Possivelmente, é o fator mais fácil e evidente de ser compreendido, independente do perfil e contexto histórico e cultural dos pesquisados. Além disso, no AQ-26B houve fusão dos fatores Medo e Percepção de Perigo (Dangerousness). Os alunos de medicina não estabeleceram uma distinção clara entre esses dois fatores, que, na realidade, traduzem traços latentes muito próximos em significado. Na versão original do questionário, a Percepção de Perigo (Dangerousness) representa o estereótipo dos portadores de transtornos mentais, imprevisíveis e violentos. Já o fator Medo está mais relacionado à reação emocional de autopreservação, provocada pelo estereótipo descrito. Como se pode observar, a reorganização de algumas questões na escala brasileira obedeceu a uma certa coerência, de acordo com a interpretação dos alunos pesquisados.

A Q1 (Eu me sentiria incomodado por José) antes no fator Anger/Irritação foi mais identificada ao fator Medo. A Q25 (Se eu fosse responsável pelo tratamento de José, iria forçá-lo a viver numa moradia protegida para pessoas com transtornos mentais) antes no fator Coersion/Coação foi mais identificada ao fator Segregação. A Q27 (Até que ponto me preocuparia com José?) antes no fator Pena foi mais identificada com o fator Desamparo, originalmente (Help/Ajuda). É possível que essa redistribuição de questões, observada a partir da análise fatorial, esteja relacionada às características da amostra: apenas alunos de medicina, aspectos da cultura brasileira, ou da 
organização dos serviços de saúde, como previstos por Wild et al. ${ }^{40}$ sobre a adaptação de instrumentos de pesquisa. Essas mudanças, além de fatores de natureza linguística e cultural, geraram a necessidade de modificação do nome de alguns fatores na versão brasileira com o intuito de se preservar maior coerência entre a distribuição das questões e seus fatores, tornando todos direcionados à estigmatização: Anger/ Irritação - Intolerância; Avoidance/Evitamento - Afastamento; Help/Ajuda - Desamparo.

Em ampla revisão sobre a mensuração do estigma relacionado à doença mental, Link ${ }^{21}$ identificou, até então, 19 estudos experimentais e 9 não experimentais em que vinhetas haviam sido utilizadas, representando 22,7\% do total de estudos realizados. Segundo esses mesmos autores, a utilização de vinhetas permite aos pesquisadores oferecer um estímulo mais elaborado do que apenas responder a perguntas sobre doenças mentais. Além disso, a possibilidade de manipulação do conteúdo das vinhetas traz a vantagem de se testar hipóteses em estudos experimentais, de forma prática e barata e em diferentes populações. É importante reconhecer também que a vinheta representa uma situação hipotética, não substituindo inteiramente a atitude de alguém na presença de uma pessoa real. Apesar dessas dificuldades, mostra-se um instrumento útil na composição de ferramentas para se mensurar o estigma.

\section{CONCLUSÃO}

O estudo em curso foi o único que verificou as propriedades psicométricas da escala AQ-27, versão em português, por meio de análise fatorial. A versão final brasileira do Questionário de Atribuição (AQ-26B) apresentou um fator a menos que a versão final de Corrigan et al. ${ }^{25}$ e dois fatores a mais que o sugerido por Brown ${ }^{28}$. A maior alteração consistiu na fusão dos fatores Medo e Percepção de Perigo (Dangerousness/Perigosidade). Apesar dessa alteração, o questionário brasileiro (AQ-26B) manteve parâmetros de validade e confiabilidade adequados, observando-se coerência com o modelo original e o gerado pelo presente estudo. Além disso, mostrou-se de fácil aplicação, podendo ser um instrumento útil para avaliar o estigma em relação à doença mental nas escolas médicas brasileiras.

\section{CONTRIBUIÇÕES INDIVIDUAIS}

Os três autores afirmam ter contribuído significativamente na concepção, no desenho e na análise dos dados do estudo. O primeiro autor escreveu o artigo na íntegra e os dois últimos autores participaram da revisão e aprovação da versão final a ser publicada.

\section{CONFLITOS DE INTERESSE}

Os autores declaram não haver quaisquer conflitos de interesse na realização deste trabalho.

\section{AGRADECIMENTOS}

O presente trabalho representa um dos objetivos da Tese de Doutorado do autor principal, desenvolvido no Programa de Pós-Graduação em Patologia, Linha de Ensino em Saúde, da Faculdade de Medicina da UFMG, e teve o apoio financeiro da Coordenação de Aperfeiçoamento de Pessoal de Nível Superior (Capes), Edital Pró-Ensino na Saúde 24/2010 (projeto № 1606/2011).

\section{REFERÊNCIAS}

1. Harris C, Barraclough B. Excess mortality of mental disorder. Br J Psychiatry. 1998;173(1): $11-53$.

2. Peluso ETP, Blay SL. Public stigma and schizophrenia in São Paulo city. Rev Bras. Psiquiatr. 2011;33(2):130-6.

3. Loch AA, Hengartner MP, Guarniero FB, Lawson FL, Wang YP, Gattaz WF, et al. 0 estigma atribuído pelos psiquiatras aos indivíduos com esquizofrenia. Rev Psiq Clín. 2011;38(5):173-7.

4. Peluso ETP, Blay SL. Community perception of mental disorders - A systematic review of Latin American and Caribbean studies. Soc Psychiatry Psychiatr Epidemiol. 2004;39(12):955-61.

5. Thornicroft G, Thornicroft G, Brohan E, Rose D, Sartorius N, Leese M; INDIGO Study Group. Global pattern of experienced and anticipated discrimination against people with schizophrenia: a cross-sectional survey. Lancet. 2009;373(9661):408-15.

6. Angermeyer MC, Mastschinger $H$. The stigma of mental ilness: efects of labelling on public attitudes towards people with mental health. Acta Psychiat Scand. 2003;108(4):304-9.

7. Corrigan PW, Lurie BD, Goldman HH, Slopen N, Medasani K, Phelan S, et al. How adolescents perceive the stigma of mental illness and alcohol abuse. Psychiatr Serv. 2005;56(5): 544-50.

8. Crisp AH, Gelder MG, Rix S, Meltzer HI, Rowlands 0J. Stigmatisation of people with menta illnesses. Br J Psychiatry. 2000;177:4-7.

9. Jones S, Howard L, Thornicroft G. 'Diagnostic overshadowing': worse physical health care for people with mental illness. Acta Psychiat Scand. 2008;118(3):169-71.

10. Rüsch N, Angermeyer MC, Corrigan PW. Mental illness stigma: concepts, consequences, and initiatives to reduce stigma. Eur Psychiatry. 2005;20(8):529-39.

11. Link BG, Cullen FT, Frank J, Wozniak JF. The social rejection of former mental patients: understanding why labels matter. Am J Sociol. 1987;92(6):1461-500.

12. Poreddi V, Thimmaiah R, Math SB. Attitudes toward people with mental illness among medical students. J Neurosci Rural Pract. 2015;6(3):349-54.

13. Kopera M, Suszek H, Bonar E, Myszka M, Gmaj B, Ilgen M, et al. Evaluating explicit and implicit stigma of mental illness in mental health professionals and medical students. Community Ment Health J. 2015;51(5):628-34.

14. Neumann E, Obliers R, Albus C. Medical students' attitudes towards mental illness: a matter of studies or personality? Psychother Psychosom Med Psychol. 2012;62(2):66-72.

15. Korszun A, Dinos S, Ahmed K, Bhui K. Medical student attitudes about mental illness: does medical-school education reduce stigma? Acad Psychiatry. 2012;36(3):197-204.

16. Llerena A, Cáceres MC, Peñas-Lledó EM. Schizophrenia stigma among medical and nursing undergraduates. Eur Psychiatry. 2002;17(5):298-9.

17. Mukherjee R, Fialho A, Wijetunge A, Checinski K, Surgenor T. The stigmatization of psychiatric illness: What attitudes do medical students and family physicians hold towards people with mental illness? Psychiatr Bull. 2002;26(1):178-81.

18. Goffman E. Stigma: notes on the management of spoiled identity. New York: Touchstone; 1963. 
19. Kassam A, Glozier N, Leese M, Henderson C, Thornicroft G. Development and responsiveness of a scale to measure clinicians' attitudes to people with mental illness (medical student version). Acta Psychiatr Scand. 2010;122(2):153-61.

20. Brohan E, Slade M, Clement S, Thornicroft $G$. Experiences of mental illness stigma, prejudice and discrimination: a review of measures. BMC Health Serv Res. 2010;10(1):80.

21. Link BG. Measuring mental illness stigma. Schizophr Bull. 2004;30(3):511-41.

22. Rodrigues CRC. Atitudes frente a doença mental: estudo transversal de uma amostra de profissionais de saúde. 1983. 148 p. Tese [Doutorado] - Faculdade de Medicina de Ribeirão Preto da USP, Ribeirão Preto; 1983

23. Pasquali L. Atitude frente ao doente mental. Rev Psicol. 1987;5(1):37-67.

24. Pasquali L. Atitude frente ao doente mental. Rev Psicol. 1987;5(2):29-71.

25. Corrigan P, Markowitz FE, Watson A, Rowan D, Kubiak MA. An attribution model of public discrimination towards persons with mental illness. J Health Soc Behav. 2003;44(2):162-79.

26. Heider F. The Psychology of interpersonal relations. New York: Wiley; 1958.

27. Weiner B. A cognitive (attribution)-emotion-action model of motivated behavior: An analysis of judgments of help-giving. J Pers Soc Psychol. 1980;39(2):186-200.

28. Brown SA. Factors and measurement of mental illness stigma: a psychometric examination of the Attribution Questionnaire. Psychiatr Rehabil J. 2008;32(2):89-94.

29. Pingani L, Forghieri M, Ferrari S, Ben-Zeev D, Artoni P, Mazzi F, et al. Stigma and discrimination toward mental illness: translation and validation of the Italian version of the Attribution Questionnaire-27 (AQ-27-I). Soc Psychiatry Psychiatr Epidemiol. 2012;47(6):993-9.

30. Pingani L, Catellani S, Del Vecchio V, Sampogna G, Ellefson SE, Rigatelli M, et al. Stigma in the context of schools: analysis of the phenomenon of stigma in a population of university students. BMC Psychiatry. 2016;6:29.
31. Sousa S, Marques A, Curral R, Queirós C. Stigmatizing attitudes in relatives of people with schizophrenia: a study using the Attribution Questionnaire AQ-27. Trends Psychiatry Psychother. 2012;34(4):186-97.

32. Reichenheim ME, Moraes $\mathrm{CL}$. Operacionalização de adaptação transcultural de instrumentos de aferição usados em epidemiologia. Rev Saúde Pública. 2007;41(4):665-73.

33. Brasil. Conselho Nacional de Saúde. Resolução n. 466, 12 de dezembro de 2012. Aprova as diretrizes e normas regulamentadoras de pesquisas envolvendo seres humanos. Diário Oficial da União, 13 jun. 2013, Seção I, p. 59.

34. Kogan JR, Shea JA. Course evaluation in medical education. Teach Teach Educ. 2007;23(3):251-64.

35. Pasquali L. Validade dos testes psicológicos: será possível reencontrar o caminho? Psic: Teor e Pesq. 2007;23(esp.):99-107.

36. Pasquali L. Psicometria: teoria dos testes na Psicologia e na Educação. 4. ed. Petrópolis: Vozes; 2011.

37. Pasquali L. Psicometria. Rev Esc Enferm USP. 2009;43(Esp):992-9.

38. Marques A, Barbosa T, Queirós C. Stigma in mental health: perceptions of students who will be future health professionals. Eur Psychiatry. 2011;26(Supp. 1):1439.

39. Barrantes FJ. 0 estigma na esquizofrenia: atitude dos profissionais da saúde mental. Tese (Reabilitaçãa Psicossocial - Perturbação Mental) - Universidade Católica Portuguesa, Porto; 2010

40. Wild D, Eremenco S, Mear I, Martin M, Houchin C, Gawlicki M, et al. Multinational trialsrecommendations on the translations required, approaches to using the same language in different countries, and the approaches to support pooling the data: the ISPOR PatientReported Outcomes Translation and Linguistic Validation Good Research Practices Task Force report. Value Health. 2009;12(4):430-40 